JOURNAL OF THE

AMERICAN MATHEMATICAL SOCIETY

Volume 16, Number 3, Pages 581-603

S 0894-0347(03)00425-9

Article electronically published on March 3, 2003

\title{
CORRELATION FUNCTION OF SCHUR PROCESS WITH APPLICATION TO LOCAL GEOMETRY OF A RANDOM 3-DIMENSIONAL YOUNG DIAGRAM
}

\author{
ANDREI OKOUNKOV AND NIKOLAI RESHETIKHIN
}

\section{INTRODUCTION}

\subsection{Schur measure and Schur process.}

1.1.1. This paper is a continuation of [16]. The Schur measure, introduced in [16], is a measure on partitions $\lambda$ that weights a partitions $\lambda$ proportionally to $s_{\lambda}(X) s_{\lambda}(Y)$, where $s_{\lambda}$ is the Schur function and $X$ and $Y$ are two sets of variables.

Here we consider a time-dependent version of the Schur measure which we call the Schur process; see Definition 2. This is a measure on sequences $\{\lambda(t)\}$ such that

$$
\operatorname{Prob}(\{\lambda(t)\}) \propto \prod \mathrm{S}^{(t)}(\lambda(t), \lambda(t+1)),
$$

where the time-dependent weight $\mathrm{S}^{(t)}(\lambda, \mu)$ is a certain generalization of a skew Schur function. It is given by a suitably regularized infinite minor of a certain Toeplitz matrix. This determinant can also be interpreted as a Karlin-McGregor nonintersection probability [12]. The distribution of each individual $\lambda(t)$ is then a Schur measure with suitable parameters.

In this paper, we consider the Schur process only in discrete time, although a continuous time formulation is also possible; see Section 2.2.11

The idea of making the Schur measure time dependent was inspired, in part, by the paper 10] which deals with some particular instances of the general concept of the Schur processes introduced here. For another development of the ideas of [10], see the paper [18] which appeared after the results of the present paper were obtained.

1.1.2. Our main interest in this paper is the correlation functions of the Schur process which, by definition, are the probabilities that the random set

$$
\mathfrak{S}(\{\lambda(t)\})=\left\{\left(t, \lambda(t)_{i}-i+\frac{1}{2}\right)\right\}, \quad t, i \in Z,
$$

contains a given set $U \subset \mathbb{Z} \times\left(\mathbb{Z}+\frac{1}{2}\right)$.

Using the same infinite wedge machinery as in [16], we prove that the correlation functions of the Schur process have a determinantal form

$$
\operatorname{Prob}(U \subset \mathfrak{S}(\{\lambda(t)\}))=\operatorname{det}\left(K\left(u_{i}, u_{j}\right)\right)_{u_{i}, u_{j} \in U},
$$

and we give an explicit contour integral representation for the correlation kernel $K$; see Theorem [1] This theorem is a generalization of Theorem 2 in [16].

Received by the editors December 8, 2001.

2000 Mathematics Subject Classification. Primary 05E05, 60G55. 


\subsection{Asymptotics.}

1.2.1. The contour integral representation for the correlation kernel is particularly convenient for asymptotic investigations. Only very elementary means, namely residue calculus and basic saddle-point analysis, are needed to derive the asymptotics.

1.2.2. We illustrate this in Section 3 by computing explicitly the correlation functions asymptotics for 3-dimensional (3D) Young diagrams in the bulk of their limit shape. The three-dimensional diagrams, also known as the plane partition, form an old subject which has received a lot of attention recently. More specifically, the measure on the 3 -dimensional diagrams $\pi$ such that

$$
\operatorname{Prob}(\pi) \propto q^{|\pi|}, \quad 0<q<1,
$$

where $|\pi|$ is the volume of $\pi$, is a particular specialization of the Schur process for which the time parameter has the meaning of an extra spatial dimension. As $q \rightarrow 1$, a typical partition, suitably scaled, approaches a limit shape which was described in [3] and can be seen in Figure 7 below. The existence of this limit shape and some of its properties were first established by A. Vershik [19], who used direct combinatorial methods.

It is well known that 3-dimensional diagrams are in bijection with certain rhombi tiling of the plane; see, for example, Section 2.3.6 below. Local statistics of random domino tilings have been the subject of intense recent studies; see, for example, [2, 4, 5, 13] and the survey [14].

The authors of [5] computed local correlations for domino tilings with periodic boundary conditions and conjectured that the same formula holds in the thermodynamic limit for domino tilings of more general regions; see Conjecture 13.5 in 5].

From the point of view of statistical mechanics, the argument behind this conjecture can be the belief that in the thermodynamic limit and away from the boundary the local correlations depend only on macroscopic parameters, such as the density of tiles of a given kind. In particular, in the case of 3-dimensional diagrams the densities of rhombi of each of the three possible kinds are uniquely fixed by the tilt of the limit shape at the point in question.

1.2.3. In this paper, we approach the local geometry of random 3-dimensional diagrams using the general exact formulas for correlation functions of the Schur process. For 3-dimensional diagrams, these general formulas specialize to contour integrals involving the quantum dilogarithm function (24); see Corollary 1 .

We compute the $q \rightarrow 1$ limit of the correlation kernel explicitly. The result turns out to be the discrete incomplete beta kernel; see Theorem 2 One can show (see Section 3.1.12) that this kernel is a specialization of the kernel of [5] when one of the parameters vanishes in agreement with Conjecture 13.5 of [5].

The incomplete beta kernel is also a bivariate generalization of the discrete sine kernel which appeared in [1] in the situation of the Plancherel specialization of the Schur measure; see also 10 .

1.3. Universality. Although we focus on one specific asymptotic problem, our methods are both very basic and completely general. They should apply, therefore, with little or no modification in a much wider variety of situations yielding the same 
or analogous results. In other words, both the methods and the results should be universal in a large class of asymptotic problems.

As explained in Section [3.2.3 this universality should be especially robust for the equal time correlations, in which case the discrete sine kernel should appear. This is parallel to the situation with random matrices $[9]$.

\section{The SCHUR PROCESS}

\subsection{Configurations.}

\subsubsection{Recall that a partition is a sequence}

$$
\lambda=\left(\lambda_{1} \geq \lambda_{2} \geq \lambda_{3} \geq \cdots \geq 0\right)
$$

of integers such that $\lambda_{i}=0$ for $i \gg 0$. The zero, or empty, partition is denoted by $\emptyset$. The book 15 is a most comprehensive reference on partitions and symmetric functions.

The Schur process is a measure on sequences

$$
\{\lambda(t)\}, \quad t \in \mathbb{Z},
$$

where each $\lambda(t)$ is a partition and $\lambda(t)=\emptyset$ for $|t| \gg 0$. We call the variable $t$ the time, even though it may have a different interpretation in applications.

2.1.2. An example of such an object is a plane partition which, by definition, is a 2-dimensional array of nonnegative numbers

$$
\pi=\left(\pi_{i j}\right), \quad i, j=1,2, \ldots,
$$

that are nonincreasing as a function of both $i$ and $j$ and such that

$$
|\pi|=\sum \pi_{i j}
$$

is finite. The plot of the function

$$
(x, y) \mapsto \pi_{\lceil x\rceil,\lceil y\rceil}, \quad x, y>0,
$$

is a 3-dimensional Young diagram with volume $|\pi|$. For example, Figure 1 shows the 3-dimensional diagram corresponding to the plane partition

$$
\pi=\left(\begin{array}{llll}
5 & 3 & 2 & 1 \\
4 & 3 & 1 & 1 \\
3 & 2 & 1 & \\
2 & 1 & &
\end{array}\right),
$$

where the entries that are not shown are zero.

We associate to $\pi$ the sequence $\{\lambda(t)\}$ of its diagonal slices, that is, the sequence of partitions

$$
\lambda(t)=\left(\pi_{i, t+i}\right), \quad i \geq \max (0,-t) .
$$

It is easy to see that a configuration $\{\lambda(t)\}$ corresponds to a plane partition if and only if it satisfies the conditions

$$
\ldots \prec \lambda(-2) \prec \lambda(-1) \prec \lambda(0) \succ \lambda(1) \succ \lambda(2) \succ \ldots,
$$

where $\lambda \succ \mu$ means that $\lambda$ and $\mu$ interlace, that is,

$$
\lambda_{1} \geq \mu_{1} \geq \lambda_{2} \geq \mu_{2} \geq \lambda_{3} \geq \ldots \text {. }
$$




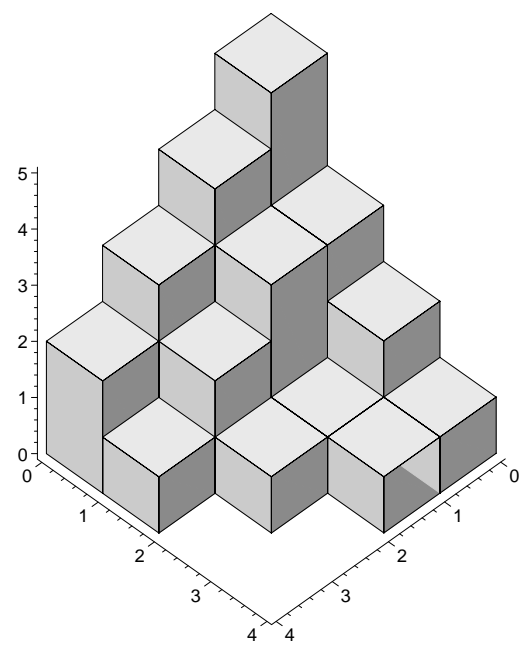

Figure 1. A 3-dimensional diagram $\pi$

In particular, the configuration $\{\lambda(t)\}$ corresponding to the diagram (21) is

$$
(2) \prec(3,1) \prec(4,2) \prec(5,3,1) \succ(3,1) \succ(2,1) \succ(1) \text {. }
$$

2.1.3. The mapping

$$
\lambda \mapsto \mathfrak{S}(\lambda)=\left\{\lambda_{i}-i+\frac{1}{2}\right\} \subset \mathbb{Z}+\frac{1}{2}
$$

is a bijection of the of the set of partitions and the set $\mathfrak{S}$ of subsets $\mathfrak{S} \subset \mathbb{Z}+\frac{1}{2}$ such that

$$
\left|\mathfrak{S} \backslash\left(\mathbb{Z}+\frac{1}{2}\right)_{<0}\right|=\left|\left(\mathbb{Z}+\frac{1}{2}\right)_{<0} \backslash \mathfrak{S}\right|<\infty
$$

The mapping

$$
\{\lambda(t)\} \mapsto \mathfrak{S}(\{\lambda(t)\})=\left\{\left(t, \lambda(t)_{i}-i+\frac{1}{2}\right)\right\} \subset \mathbb{Z} \times\left(\mathbb{Z}+\frac{1}{2}\right)
$$

identifies the configurations of the Schur process with certain subsets of $\mathbb{Z} \times\left(\mathbb{Z}+\frac{1}{2}\right)$. In other words, the mapping (5) makes the Schur process a random point field on $\mathbb{Z} \times\left(\mathbb{Z}+\frac{1}{2}\right)$.

For example, the subset $\mathfrak{S}(\{\lambda(t)\})$ corresponding to the 3 -dimensional diagram from Figure 1 is shown in Figure 2 One can also visualize $\mathfrak{S}(\{\lambda(t)\})$ as a collection of nonintersecting paths as in Figure 2

\subsection{Probabilities.}

2.2.1. The Schur process, the formal definition of which is given in Definition 2 below, is a measure on sequences $\{\lambda(t)\}$ such that

$$
\operatorname{Prob}(\{\lambda(t)\}) \propto \prod_{t \in \mathbb{Z}} \mathrm{S}^{(t)}(\lambda(t), \lambda(t+1)),
$$

where $\mathrm{S}^{(t)}(\mu, \lambda)$ is a certain time-dependent transition weight between the partition $\mu$ and $\lambda$ which will be defined presently. 


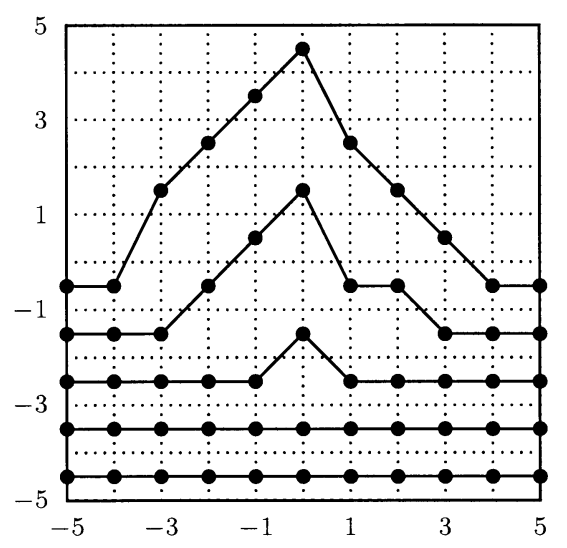

Figure 2. Point field and nonintersecting paths corresponding to the diagram in Figure 1

The coefficient $\mathbf{S}^{(t)}(\mu, \lambda)$ is a suitably regularized infinite minor of a certain Toeplitz matrix. It can be viewed as a generalization of the Jacobi-Trudy determinant for a skew Schur function or as a form of Karlin-McGregor nonintersection probability [12].

\subsubsection{Let a function}

$$
\phi(z)=\sum_{k \in \mathbb{Z}} \phi_{k} z^{k}
$$

be nonvanishing on the unit circle $|z|=1$ with winding number 0 and geometric mean 1 . These two conditions mean that $\log \phi$ is a well defined function with mean 0 on the unit circle. For simplicity, we additionally assume that $\phi$ is analytic in some neighborhood of the unit circle. Many of the results below hold under weaker assumptions on $\phi$ as can be seen, for example, by an approximation argument. We will not pursue here the greatest analytic generality.

\subsubsection{Given two subsets}

$$
X=\left\{x_{i}\right\}, \quad Y=\left\{y_{i}\right\} \in \mathfrak{S},
$$

we wish to assign a meaning to the following infinite determinant:

$$
\operatorname{det}\left(\phi_{y_{i}-x_{j}}\right) \text {. }
$$

We will see that, even though there is no canonical way to evaluate this determinant, different regularizations differ by a constant which depends only on $\phi$ and not on $X$ and $Y$.

Since our goal is to define probabilities only up to a constant factor, it is clear that different regularizations lead to the same random process.

2.2.4. The function $\phi$ admits a Wiener-Hopf factorization of the form

$$
\phi(z)=\phi^{+}(z) \phi^{-}(z)
$$

where the functions

$$
\phi^{ \pm}(z)=1+\sum_{k \in \pm \mathbb{N}} \phi_{k}^{ \pm} z^{k}
$$


are analytic and nonvanishing in some neighborhood of the interior (resp., exterior) of the unit disk.

There is a special case when the meaning of (6) in unambiguous; namely, if $\phi^{-}(z)=1\left(\right.$ or $\left.\phi^{+}(z)=1\right)$, then the matrix is almost unitriangular and the determinant in (6) is essentially a finite determinant. This determinant is then a Jacobi-Trudy determinant for a skew Schur function

$$
\operatorname{det}\left(\phi_{\lambda_{i}-\mu_{j}+j-i}^{+}\right)=s_{\lambda / \mu}\left(\phi^{+}\right)
$$

where $s_{\lambda / \mu}\left(\phi^{+}\right)$is the skew Schur function $s_{\lambda / \mu}$ specialized so that

$$
h_{k}=\phi_{k}^{+}
$$

where $h_{k}$ are the complete homogeneous symmetric functions. Note that

$$
s_{\lambda / \mu}\left(\phi^{+}\right)=0, \quad \mu \not \subset \lambda .
$$

2.2.5.

Definition 1. We define the transition weight by the following formula

$$
\mathrm{S}_{\phi}(\mu, \lambda)=\sum_{\nu} s_{\mu / \nu}\left(\phi^{-}\right) s_{\lambda / \nu}\left(\phi^{+}\right),
$$

where $s_{\lambda / \nu}\left(\phi^{+}\right)$is defined by (17) and $s_{\mu / \nu}\left(\phi^{-}\right)$is the skew Schur function $s_{\mu / \nu}$ specialized so that

$$
h_{k}=\phi_{-k}^{-} .
$$

Note that if the determinant $\operatorname{det}\left(\phi_{\lambda_{i}-\mu_{j}+j-i}\right)$ were unambiguously defined and satisfied the Cauchy-Binet formula, then it would equal (9) because

$$
\left(\phi_{i-j}\right)=\left(\phi_{i-j}^{+}\right)\left(\phi_{i-j}^{-}\right) \text {. }
$$

Also note that because of (8) the sum in (9) is finite; namely, it ranges over all $\nu$ such that $\nu \subset \mu, \lambda$.

2.2.6. In what follows, we will assume that the reader is familiar with the basics of the infinite wedge formalism; see, for example, Chapter 14 of the book [1] by V. Kac. An introductory account of this formalism, together with some probablistic applications, can be found in the lectures [17. We will use the notation conventions of the appendix to 16 summarized for the reader's convenience in the appendix to this paper.

Consider the following vertex operators

$$
\Gamma_{ \pm}(\phi)=\exp \left(\sum_{k=1}^{\infty}(\log \phi)_{\mp k} \alpha_{ \pm k}\right)
$$

where $(\log \phi)_{k}$ denotes the coefficient of $z^{k}$ in the Laurent expansion of $\log \phi(z)$. In particular, if the algebra of symmetric functions is specialized as in (7), then

$$
(\log \phi)_{k}=\frac{p_{k}}{k}, \quad k=1,2,3, \ldots,
$$

where $p_{k}$ is the $k$ th power-sum symmetric function.

It is well known (see, for example, Exercise 14.26 in [11]) that the matrix coefficient of the operators $\Gamma_{ \pm}$are the skew Schur functions; namely,

$$
\left(\Gamma_{-}(\phi) v_{\mu}, v_{\lambda}\right)=s_{\lambda / \mu}\left(\phi^{+}\right)
$$


It follows that

$$
\mathrm{S}_{\phi}(\mu, \lambda)=\left(\Gamma_{-}(\phi) \Gamma_{+}(\phi) v_{\mu}, v_{\lambda}\right)
$$

It is clear from the commutation relation

$$
\Gamma_{+}(\phi) \Gamma_{-}(\phi)=e^{\sum k(\log \phi)_{k}(\log \phi)_{-k}} \Gamma_{-}(\phi) \Gamma_{+}(\phi)
$$

that different ordering prescriptions in (10), which produce different regularizations of (6), all differ by a constant independent of $\mu$ and $\lambda$.

2.2.7. Now suppose that for any half-integer $m \in \mathbb{Z}+\frac{1}{2}$ we choose, independently, a function

$$
\phi[m](z)=\sum_{k \in \mathbb{Z}} \phi_{k}[m] z^{k}
$$

as above so that the series

$$
\sum_{m \in \mathbb{Z}} \log \phi[m](z)
$$

converges absolutely and uniformly in some neighborhood of the unit disk. This assumption is convenient but can be weakened. The functions $\phi[m]$ will be the parameters of the Schur process.

Definition 2. The probabilities of the Schur process are given by

$$
\operatorname{Prob}(\{\lambda(t)\})=\frac{1}{Z} \prod_{m \in \mathbb{Z}+1 / 2} \mathrm{~S}_{\phi[m]}\left(\lambda\left(m-\frac{1}{2}\right), \lambda\left(m+\frac{1}{2}\right)\right),
$$

where the transition weight $\mathrm{S}_{\phi}$ is defined in Definition 1 and $Z$ is the normalizing factor (partition function)

$$
Z=\sum_{\{\lambda(t)\}} \prod_{m \in \mathbb{Z}+1 / 2} \mathrm{~S}_{\phi[m]}\left(\lambda\left(m-\frac{1}{2}\right), \lambda\left(m+\frac{1}{2}\right)\right) .
$$

2.2.8. It follows from (10) that $Z$ is given by the following matrix coefficient

$$
Z=\left(\prod_{m \in \mathbb{Z}+1 / 2} \Gamma_{-}(\phi[m]) \Gamma_{+}(\phi[m]) v_{\emptyset}, v_{\emptyset}\right)
$$

where $\overleftarrow{\prod}$ denotes the time-ordered product, that is, the product in which operators are ordered from right to left in increasing time order.

Using (11) and the following consequence of (43)

$$
\Gamma_{+}(\phi) v_{\emptyset}=v_{\emptyset},
$$

we compute the matrix coefficient (12) as follows

$$
Z=\exp \left(\sum_{m_{1}<m_{2}} \sum_{k} k\left(\log \phi\left[m_{1}\right]\right)_{k}\left(\log \phi\left[m_{2}\right]\right)_{-k}\right) .
$$

Our growth assumptions on the functions $\phi[m]$ ensure the convergence of $Z$. 
2.2.9. It is clear from the vertex-operator description that

$$
\operatorname{Prob}(\lambda(t)=\mu) \propto s_{\mu}\left(\prod_{m<t} \phi^{+}[m]\right) s_{\mu}\left(\prod_{m>t} \phi^{-}[m]\right),
$$

where, for example, the first factor is the image of the Schur function under the specialization that sets $h_{k}$ to the coefficient of $z^{k}$ in the product of $\phi^{+}[m](z)$ over $m<t$.

This means that the distribution of each individual $\lambda(t)$ is a Schur measure [16] with parameters (14).

2.2.10. More generally, the restriction of the Schur process to any subset of times is again a Schur process with suitably modified parameters. Specifically, let a subset

$$
\left\{t_{k}\right\} \subset \mathbb{Z}, \quad k \in \mathbb{Z},
$$

be given and consider the restriction of the Schur process to this set; that is, consider the process

$$
\{\tilde{\lambda}(k)\}=\left\{\lambda\left(t_{k}\right)\right\} .
$$

It follows from the vertex operator description that this is again a Schur process with parameters

$$
\widetilde{\phi}[l]=\prod_{t_{l-\frac{1}{2}}<m<t_{l+\frac{1}{2}}} \phi[m], \quad l, m \in \mathbb{Z}+\frac{1}{2} .
$$

The case of a finite set $\left\{t_{k}\right\}$ is completely analogous and can be dealt with formally by allowing infinite values of $t_{k}$ 's.

2.2.11. The restriction property from Section 2.2.10 forms a natural basis for considering the Schur process in continuous time.

For this we need a function $\mathcal{L}(z, s)$ of a continuous variable $s \in \mathbb{R}$ which will play the role of the density of $\log \phi$. The restriction $\left\{\lambda\left(t_{k}\right)\right\}$ of the process $\lambda(t), t \in \mathbb{R}$, to any discrete set of times $\left\{t_{k}\right\}$ is the Schur process with parameters

$$
\phi[m](z)=\exp \left(\int_{t_{m-\frac{1}{2}}}^{t_{m+\frac{1}{2}}} \mathcal{L}(z, s) d s\right), \quad m \in \mathbb{Z}+\frac{1}{2} .
$$

2.2.12. Let $q \in(0,1)$ and consider the probability measure $\mathfrak{M}_{q}$ on the set of all 3 -dimensional diagrams such that

$$
\operatorname{Prob}(\pi) \propto q^{|\pi|} .
$$

We claim that there exists a particular choice of the parameters of the Schur process which yield this measure under the correspondence (3). Concretely, set

$$
\phi_{3 D}[m](z)=\left\{\begin{array}{ll}
\left(1-q^{|m|} z\right)^{-1}, & m<0, \\
\left(1-q^{|m|} z^{-1}\right)^{-1}, & m>0,
\end{array} \quad m \in \mathbb{Z}+\frac{1}{2} .\right.
$$

It is well known that if the algebra of the symmetric functions is specialized so that

$$
h_{k}=c^{k}, \quad k=1,2, \ldots,
$$

for some constant $c$, then

$$
s_{\lambda / \mu}= \begin{cases}c^{|\lambda|-|\mu|}, & \mu \prec \lambda, \\ 0, & \mu \nprec \lambda .\end{cases}
$$


Therefore, we have

$$
\begin{aligned}
& \mathrm{S}_{\phi_{3 D}[m]}\left(\lambda\left(m-\frac{1}{2}\right), \lambda\left(m+\frac{1}{2}\right)\right)= \\
& \qquad \begin{cases}q^{m\left(\left|\lambda\left(m-\frac{1}{2}\right)\right|-\left|\lambda\left(m+\frac{1}{2}\right)\right|\right)}, & m<0, \lambda\left(m-\frac{1}{2}\right) \prec \lambda\left(m+\frac{1}{2}\right) \quad \text { or } \\
0, & m>0, \lambda\left(m-\frac{1}{2}\right) \succ \lambda\left(m+\frac{1}{2}\right),\end{cases}
\end{aligned}
$$

It follows that for the specialization (15) the Schur process is supported on configurations of the shape (4) and the weight of a configuration (4) is proportional to

$$
q^{\sum|\lambda(t)|}=q^{|\pi|} .
$$

In particular, the partition function $Z$ becomes

$$
\begin{aligned}
Z_{3 D}=\exp \left(\sum_{m_{1}, m_{2}=1 / 2}^{\infty} \sum_{k} \frac{q^{k\left(m_{1}+m_{2}\right)}}{k}\right) & = \\
& \prod_{m_{1}, m_{2}}\left(1-q^{m_{1}+m_{2}}\right)^{-1}=\prod_{n=1}^{\infty}\left(1-q^{n}\right)^{-n},
\end{aligned}
$$

which is the well-known generating function, due to McMahon, for 3-dimensional diagrams.

\subsection{Correlation functions.}

2.3.1.

Definition 3. Given a subset $U \subset \mathbb{Z} \times\left(\mathbb{Z}+\frac{1}{2}\right)$, define the corresponding correlation function by

$$
\rho(U)=\operatorname{Prob}(U \subset \mathfrak{S}(\{\lambda(t)\})) .
$$

These correlation functions depend on the parameters $\phi[m]$ of the Schur process.

In this section we show that

$$
\rho(U)=\operatorname{det}\left(K\left(u_{i}, u_{j}\right)\right)_{u_{i}, u_{j} \in U},
$$

for a certain kernel $K$ which will be computed explicitly.

\subsubsection{Suppose that}

$$
U=\left\{u_{1}, \ldots, u_{n}\right\}, \quad u_{i}=\left(t_{i}, x_{i}\right) \in \mathbb{Z} \times\left(\mathbb{Z}+\frac{1}{2}\right),
$$

and the points $u_{i}$ are ordered so that

$$
t_{1} \leq t_{2} \leq t_{3} \cdots \leq t_{n} .
$$

For convenience, we set $t_{0}=-\infty$. From (10) and (41) it is clear that

$$
\rho(U)=\frac{1}{Z}\left(R_{U} v_{\emptyset}, v_{\emptyset}\right) \text {, }
$$

where $R_{U}$ is the operator

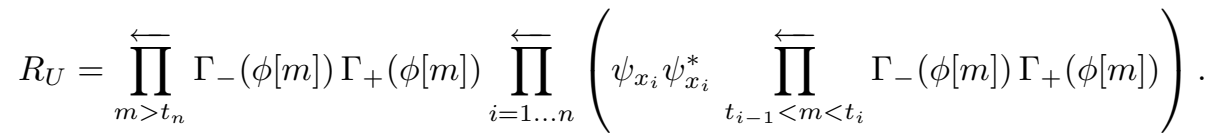


2.3.3. Define the operator

$$
\Psi_{x}(t)=\operatorname{Ad}\left(\prod_{m>t} \Gamma_{+}(\phi[m]) \prod_{m<t} \Gamma_{-}(\phi[m])^{-1}\right) \cdot \psi_{x},
$$

where Ad denotes the action by conjugation, and define the operator $\Psi_{x}^{*}(t)$ similarly. Note that the ordering of the vertex operators inside the Ad symbol is immaterial because the vertex operators commute up to a central element.

It follows from (17), (13), and (12) that

$$
\rho(U)=\left(\overleftarrow{\prod} \Psi_{x_{i}}\left(t_{i}\right) \Psi_{x_{i}}^{*}\left(t_{i}\right) v_{\emptyset}, v_{\emptyset}\right)
$$

Now we apply the Wick formula in the following form:

Lemma 1 (Wick formula). Let $A_{i}=\sum_{k} a_{i, k} \psi_{k}$ and $A_{i}^{*}=\sum_{k} a_{i, k}^{*} \psi_{k}^{*}$. Then

$$
\left(\prod A_{i} A_{i}^{*} v_{\emptyset}, v_{\emptyset}\right)=\operatorname{det}\left(K_{A}(i, j)\right)
$$

where

$$
K_{A}(i, j)= \begin{cases}\left(A_{i} A_{j}^{*} v_{\emptyset}, v_{\emptyset}\right), & i \geq j, \\ -\left(A_{j}^{*} A_{i} v_{\emptyset}, v_{\emptyset}\right), & i<j .\end{cases}
$$

Proof. Both sides of (18) are linear in $a_{i, k}$ and $a_{i, k}^{*}$; therefore it suffices to verify (18) for some linear basis in the space of possible $A_{i}$ 's and $A_{j}^{*}$ 's. A convenient linear basis is formed by the series (19) as the parameter $z$ varies. Using the canonical anticommutation relation satisfied by $\psi(z)$ and $\psi^{*}(z)$, one then verifies (18) directly.

We obtain the formula (16) with

$$
K\left(\left(t_{1}, x_{1}\right),\left(t_{2}, x_{2}\right)\right)= \begin{cases}\left(\Psi_{x_{1}}\left(t_{1}\right) \Psi_{x_{2}}^{*}\left(t_{2}\right) v_{\emptyset}, v_{\emptyset}\right), & t_{1} \geq t_{2}, \\ -\left(\Psi_{x_{2}}^{*}\left(t_{2}\right) \Psi_{x_{1}}\left(t_{1}\right) v_{\emptyset}, v_{\emptyset}\right), & t_{1}<t_{2} .\end{cases}
$$

Note that for $t_{1} \neq t_{2}$ the operators $\Psi_{x_{1}}\left(t_{1}\right)$ and $\Psi_{x_{2}}^{*}\left(t_{2}\right)$ do not, in general, anticommute so the time ordering is important. However, for $t_{1}=t_{2}$ and $x_{1} \neq x_{2}$, these operators do anticommute, so at equal time the ordering is immaterial.

2.3.4. A convenient generating function for the kernel $K$ can be obtained as follows. Set

$$
\psi(z)=\sum_{k \in \mathbb{Z}+1 / 2} z^{k} \psi_{k}, \quad \psi^{*}(z)=\sum_{k \in \mathbb{Z}+1 / 2} z^{-k} \psi_{k}^{*}
$$

Set also

$$
\Psi(t, z)=\operatorname{Ad}\left(\prod_{m>t} \Gamma_{+}(\phi[m]) \prod_{m<t} \Gamma_{-}(\phi[m])^{-1}\right) \cdot \psi(z),
$$

and define $\Psi^{*}(t, z)$ similarly.

We have from (42)

$$
\begin{aligned}
& \operatorname{Ad}\left(\Gamma_{ \pm}(\phi)\right) \cdot \psi(z)=\phi^{\mp}\left(z^{-1}\right) \psi(z), \\
& \operatorname{Ad}\left(\Gamma_{ \pm}(\phi)\right) \cdot \psi^{*}(z)=\phi^{\mp}\left(z^{-1}\right)^{-1} \psi(z)
\end{aligned}
$$

and therefore

$$
\Psi(t, z)=\Phi(t, z) \psi(z), \quad \Psi^{*}(t, z)=\Phi(t, z)^{-1} \psi(z)
$$


where

$$
\Phi(t, z)=\frac{\prod_{m>t} \phi^{-}[m]\left(z^{-1}\right)}{\prod_{m<t} \phi^{+}[m]\left(z^{-1}\right)} .
$$

Finally, it is obvious from the definitions that

$$
\begin{aligned}
& \left(\psi(z) \psi^{*}(w) v_{\emptyset}, v_{\emptyset}\right)=\frac{\sqrt{z w}}{z-w}, \quad|z|>|w|, \\
& -\left(\psi^{*}(w) \psi(z) v_{\emptyset}, v_{\emptyset}\right)=\frac{\sqrt{z w}}{z-w}, \quad|z|<|w| .
\end{aligned}
$$

Putting it all together, we obtain the following

Theorem 1. We have

$$
\rho(U)=\operatorname{det}\left(K\left(u_{i}, u_{j}\right)\right)_{u_{i}, u_{j} \in U},
$$

where the kernel $K$ is determined by the generating function

$$
\begin{aligned}
\mathrm{K}_{t_{1}, t_{2}}(z, w) & =\sum_{x_{1}, x_{2} \in \mathbb{Z}+\frac{1}{2}} z^{x_{1}} w^{-x_{2}} K\left(\left(t_{1}, x_{1}\right),\left(t_{2}, x_{2}\right)\right), \\
& =\frac{\sqrt{z w}}{z-w} \frac{\Phi\left(t_{1}, z\right)}{\Phi\left(t_{2}, w\right)} .
\end{aligned}
$$

Here the function $\Phi(t, z)$ is defined by (20), and (21) is the expansion of (22) in the region $|z|>|w|$ if $t_{1} \geq t_{2}$ and $|z|<|w|$ if $t_{1}<t_{2}$.

2.3.5. In the special case of the measure $\mathfrak{M}_{q}$ on the 3 -dimensional diagrams the function (20) specializes to the following function

$$
\Phi_{3 D}(t, z)=\frac{\prod_{m>\max (0,-t)}\left(1-q^{m} / z\right)}{\prod_{m>\max (0, t)}\left(1-q^{m} z\right)}, \quad m \in \mathbb{Z}+\frac{1}{2} .
$$

Consider the function

$$
(z ; q)_{\infty}=\prod_{n=0}^{\infty}\left(1-q^{n} z\right) .
$$

For various reasons, in particular because of the relation (28), this function is sometimes called the quantum dilogarithm function [6].

It is clear that the function (23) has the following expression in terms of the quantum dilogarithm:

$$
\Phi_{3 D}(t, z)= \begin{cases}\frac{\left(q^{1 / 2} / z ; q\right)_{\infty}}{\left(q^{1 / 2+t} z ; q\right)_{\infty}}, & t \geq 0 \\ \frac{\left(q^{1 / 2-t} / z ; q\right)_{\infty}}{\left(q^{1 / 2} z ; q\right)_{\infty}}, & t \leq 0\end{cases}
$$

2.3.6. In order to make a better connection with the geometry of 3-dimensional diagrams, let us introduce a different encoding of diagrams by subsets in the plane. Given a plane partition

$$
\pi=\left(\pi_{i j}\right), \quad i, j=1,2, \ldots
$$

we set

$$
\widetilde{\mathfrak{S}}(\pi)=\left\{\left(j-i, \pi_{i j}-(i+j-1) / 2\right)\right\}, \quad i, j=1,2, \ldots
$$


There is a well-known correspondence between 3-dimensional diagrams and tilings of the plane by rhombi. Namely, the tiles are the images of the faces of the 3dimensional diagram under the projection

$$
(x, y, z) \mapsto(t, h)=(y-x, z-(x+y) / 2) .
$$

The tiling corresponding to the diagram in Figure 1 is shown in Figure 3 .

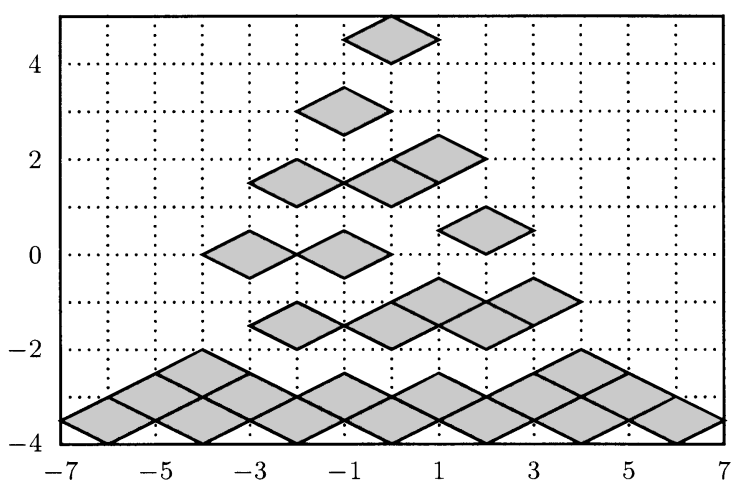

Figure 3. Horizontal tiles of the tiling corresponding to the diagram in Figure 1

It is clear that under this correspondence the horizontal faces of a 3-dimensional diagram are mapped to the horizontal tiles and that the positions of the horizontal tiles uniquely determine the tiling and the diagram $\pi$. The set

$$
\widetilde{\mathfrak{S}}(\pi) \subset \mathbb{Z} \times \frac{1}{2} \mathbb{Z}
$$

is precisely the set of the centers of the horizontal tiles. It is also clear that if $\{\lambda(t)\}$ corresponds to the diagram $\pi$, then

$$
(t, h) \in \widetilde{\mathfrak{S}}(\pi) \Leftrightarrow(t, x+|t| / 2) \in \mathfrak{S}(\{\lambda(t)\}) .
$$

Theorem 1 specializes, therefore, to the following statement:

Corollary 1. For any set $\left\{\left(t_{i}, h_{i}\right)\right\}$, we have

$$
\operatorname{Prob}\left(\left\{\left(t_{i}, h_{i}\right)\right\} \subset \widetilde{\mathfrak{S}}(\pi)\right)=\operatorname{det}\left[K_{3 D}\left(\left(t_{i}, h_{i}\right),\left(t_{j}, h_{j}\right)\right)\right]
$$

where the kernel $K_{3 D}$ is given by the formula

$$
\begin{aligned}
& K_{3 D}\left(\left(t_{1}, h_{1}\right),\left(t_{2}, h_{2}\right)\right)= \\
& \quad \frac{1}{(2 \pi i)^{2}} \int_{|z|=1 \pm \epsilon} \int_{|w|=1 \mp \epsilon} \frac{1}{z-w} \frac{\Phi_{3 D}\left(t_{1}, z\right)}{\Phi_{3 D}\left(t_{2}, w\right)} \frac{d z d w}{z^{h_{1}+\frac{\left|t_{1}\right|+1}{2}} w^{-h_{2}-\frac{\left|t_{2}\right|-1}{2}}} .
\end{aligned}
$$

Here the function $\Phi_{3 D}(t, z)$ is defined by (23), $0<\epsilon \ll 1$, and one picks the plus sign if $t_{1} \geq t_{2}$ and the negative sign otherwise. 


\section{Asymptotics}

\subsection{The local shape of a large 3-dimensional diagram.}

3.1.1. Our goal in this section is to illustrate how suitable the formula (22) is for asymptotic analysis. In order to be specific, we work out one concrete example, namely, the local shape of a 3-dimensional diagram distributed according to the measure $\mathfrak{M}_{q}$ as $q \rightarrow 1$. Our computations, however, will be of a very abstract and general nature and applicable to a much wider variety of specialization.

The reader will notice that passage to the asymptotics in (22) is so straightforward that even the saddle-point analysis is needed in only a very weak form, namely, as the statement that

$$
\int_{\gamma} e^{M S(x)} d x \rightarrow 0, \quad M \rightarrow+\infty
$$

provided the function $S(x)$ is smooth and $\Re S(x)<0$ for all but finitely many points $x \in \gamma$.

3.1.2. Let $q=e^{-r}$ and $r \rightarrow+0$. We begin with the following

Lemma 2. We have the following convergence in probability

$$
r^{3}|\pi| \rightarrow 2 \zeta(3), \quad r \rightarrow+0,
$$

where $|\pi|$ is the volume of a 3 -dimensional diagram $\pi$ sampled from the measure $\mathfrak{M}_{q}$.

Proof. First consider the expectation of $|\pi|$

$$
\mathrm{E}|\pi|=\frac{q \frac{d}{d q} Z_{3 D}}{Z_{3 D}}=\sum_{n \geq 1} \frac{n^{2} q^{n}}{1-q^{n}}=\sum_{n, k \geq 1} n^{2} q^{n k}=\sum_{k} \frac{q^{k}\left(1+q^{k}\right)}{\left(1-q^{k}\right)^{3}} \sim \frac{2 \zeta(3)}{r^{3}} .
$$

Similarly, the variance of $|\pi|$ behaves like

$$
\operatorname{Var}|\pi|=q \frac{d}{d q} \mathrm{E}|\pi|=o\left(r^{-6}\right),
$$

whence $\operatorname{Var}\left(r^{3}|\pi|\right) \rightarrow 0$, which concludes the proof.

3.1.3. It follows that as $r \rightarrow+0$, the typical 3-dimensional diagram $\pi$, scaled by $r$ in all directions, approaches the suitably scaled limit shape for typical 3-dimensional diagrams of a large volume described in 3 . Below we will also see this limit shape appear from our calculations.

We are interested in the $r \rightarrow+0$ limiting local structure of $\pi$ in the neighborhood of various points in the limit shape. In other words, we are interested in the limit of the kernel (26) as

$$
r t_{i} \rightarrow \tau, \quad r h_{i} \rightarrow \chi,
$$

where the variables $\tau$ and $\chi$ describe the global position on the limit shape, in such a way that the relative distances

$$
\Delta t=t_{1}-t_{2}, \quad \Delta h=h_{1}-h_{2}
$$

remain fixed. This limit is easy to obtain by a combination of residue calculus with saddle-point argument. Since the measure $\mathfrak{M}_{q}$ is obviously symmetric with respect to the reflection $t \mapsto-t$, we can without loss of generality assume that $\tau \geq 0$ in our computations. 
3.1.4. We have the following $r \rightarrow+0$ asymptotics:

$$
\ln (z ; q)_{\infty} \sim r^{-1} \int_{0}^{z} \frac{\ln (1-w)}{w} d w=-r^{-1} \operatorname{dilog}(1-z),
$$

where

$$
\operatorname{dilog}(1-z)=\sum_{n} \frac{z^{n}}{n^{2}}, \quad|z| \leq 1,
$$

analytically continued with a cut along $(1,+\infty)$.

Introduce the following function

$$
S(z ; \tau, \chi)=-(\tau / 2+\chi) \ln z-\operatorname{dilog}(1-1 / z)+\operatorname{dilog}\left(1-e^{-\tau} z\right)
$$

and recall that we made the assumption that $\tau \geq 0$. The function $S(z ; \tau, \chi)$ is analytic in the complex plane with cuts along $(0,1)$ and $\left(e^{\tau},+\infty\right)$.

As $r \rightarrow+0$, the exponentially large term in the integrand in (26) is

$$
\exp \left(\frac{1}{r}(S(z ; \tau, \chi)-S(w ; \tau, \chi))\right) .
$$

The saddle-point method suggests, therefore, to look at the critical points of the function $S(z ; \tau, \chi)$. Since

$$
z \frac{d}{d z} S(z ; \tau, \chi)=-\tau / 2-\chi-\log (1-1 / z)\left(1-e^{-\tau} z\right),
$$

the critical points of $S$ are the roots of the quadratic polynomial

$$
(1-1 / z)\left(1-e^{-\tau} z\right)=e^{-\tau / 2-\chi} .
$$

The two roots of this polynomial are complex conjugate if

$$
\left|e^{\tau / 2}+e^{-\tau / 2}-e^{-\chi}\right|<2,
$$

which can be expressed equivalently as

$$
-2 \ln \left(2 \cosh \frac{\tau}{4}\right)<\chi<-2 \ln \left(2 \sinh \frac{\tau}{4}\right) .
$$

In the case when the roots are complex conjugate, they lie on the circle

$$
\gamma=\left\{|z|=e^{\tau / 2}\right\} .
$$

As we shall see below, the inequality (30) describes precisely the possible values of $(\tau, \chi)$ that correspond to the bulk of the limit shape.

3.1.5. The following elementary properties of the function $S(z ; \tau, \chi)$

$$
\begin{gathered}
S(\bar{z} ; \tau, \chi)=\overline{S(z ; \tau, \chi)}, \\
S(z ; \tau, \chi)+S\left(e^{\tau} / z ; \tau, \chi\right)=-(\tau / 2+\chi) \tau,
\end{gathered}
$$

imply that on the circle $\gamma$ the real part of $S$ is constant; namely,

$$
\Re S(z ; \tau, \chi)=-(\tau / 2+\chi) \tau / 2, \quad z \in \gamma .
$$

On $\gamma$ we also have

$$
z \frac{d}{d z} S(z ; \tau, \chi)=3 \tau / 2-\chi-\ln \left|e^{\tau}-z\right|^{2}, \quad z \in \gamma .
$$

From this it is clear that when the critical points of $S$ are complex conjugate, they are the points of intersection of the two circles

$$
\left\{z_{c}, \bar{z}_{c}\right\}=\left\{|z|=e^{\tau / 2}\right\} \cap\left\{\left|z-e^{\tau}\right|=e^{3 \tau / 4-\chi / 2}\right\} .
$$




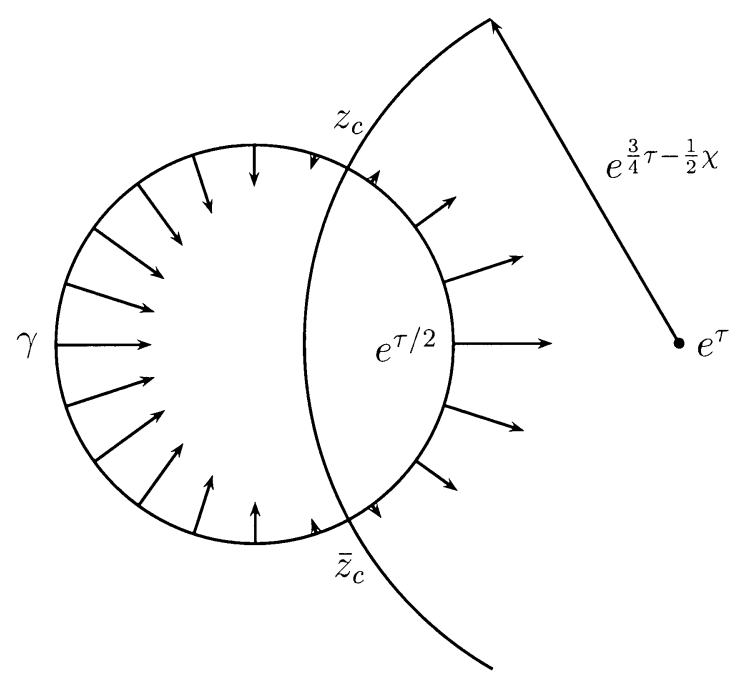

FiguRE 4. Gradient of $\Re S(z)$ on the circle $\gamma=\left\{|z|=e^{\tau / 2}\right\}$

This is illustrated in Figure 4 which also shows the vector field

$$
\nabla(\Re S(z ; \tau, \chi))=\frac{z^{2}}{e^{\tau}} \frac{d}{d z} S(z), \quad z \in \gamma .
$$

3.1.6. Now we are prepared to do the asymptotics in (26). We can deform the contours of the integration in (26) as follows

$$
K_{3 D}\left(\left(t_{i}, h_{i}\right),\left(t_{j}, h_{j}\right)\right)=\frac{1}{(2 \pi i)^{2}} \int_{(1 \pm \epsilon) \gamma} d z \int_{(1 \mp \epsilon) \gamma} d w \quad \ldots
$$

where the dots stand for the same integrand as in (26), $0<\epsilon \ll 1$, and we pick the plus sign if $t_{1} \geq t_{2}$ and the negative sign otherwise.

Now we define the contours $\gamma_{>}, \gamma_{<}, \gamma_{+}, \gamma_{-}$. This definition will be illustrated by Figure 5. The contour $\gamma_{>}$is the circle $|z|=e^{\tau / 2}$ slightly deformed in the direction of the gradient of $\Re S$; see Figure 4. Similarly, the contour $\gamma_{<}$is the same circle $|z|=e^{\tau / 2}$ slightly pushed in the opposite direction. The contours $\gamma_{ \pm}$are the arcs of the circle $|z|=e^{\tau / 2}$ between $\bar{z}_{c}$ and $z_{c}$, oriented toward $z_{c}$.

Deforming the contours and picking the residue at $z=w$, we obtain

$$
K_{3 D}\left(\left(t_{i}, h_{i}\right),\left(t_{j}, h_{j}\right)\right)=\int^{(1)}+\int^{(2)},
$$

where

$$
\int^{(1)}=\frac{1}{(2 \pi i)^{2}} \int_{\gamma_{<}} d z \int_{\gamma_{>}} d w \quad \cdots
$$

with the same integrand in as in (26) and

$$
\int^{(2)}=\frac{1}{2 \pi i} \int_{\gamma_{ \pm}} \frac{\left(q^{1 / 2+t_{2}} w ; q\right)_{\infty}}{\left(q^{1 / 2+t_{1}} w ; q\right)_{\infty}} \frac{d w}{w^{\Delta h+\Delta t / 2+1}}
$$




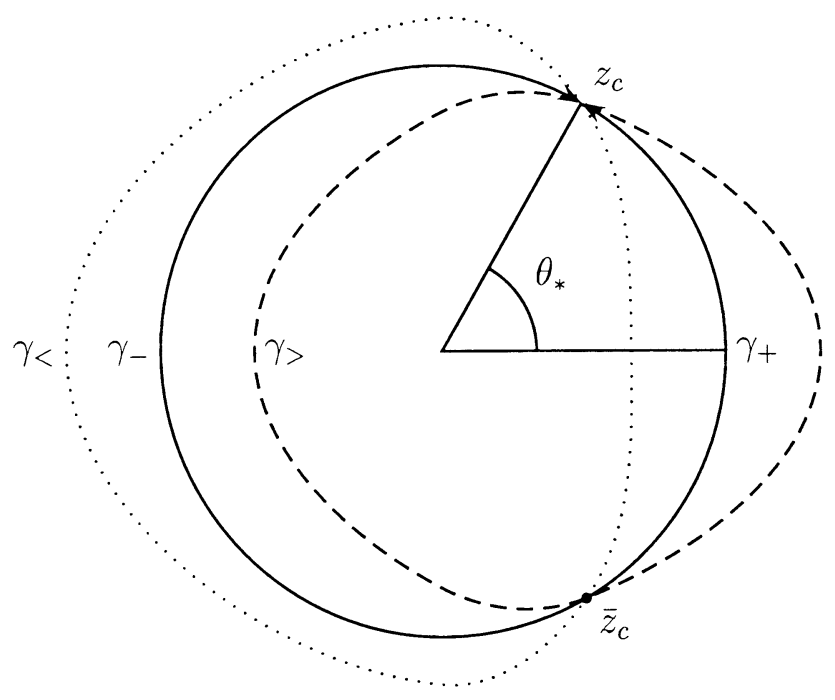

Figure 5. Contours $\gamma_{>}$(dashed), $\gamma_{<}$(dotted), and $\gamma_{ \pm}$

where we choose $\gamma_{+}$if $t_{1} \geq t_{2}$ and $\gamma_{-}$otherwise. As we shall see momentarily,

$$
\int^{(1)} \rightarrow 0, \quad r \rightarrow+0
$$

while $\int^{(2)}$ has a simple limit.

3.1.7. It is obvious that

$$
\int^{(2)} \rightarrow \frac{1}{2 \pi i} \int_{\gamma_{ \pm}}\left(1-e^{-\tau} w\right)^{\Delta t} \frac{d w}{w^{\Delta h+\Delta t / 2+1}}, \quad r \rightarrow+0 .
$$

The change of variables $w=e^{\tau} w^{\prime}$ makes this integral a standard incomplete beta function integral:

$$
\int^{(2)} \rightarrow \frac{e^{-\tau(\Delta h+\Delta t / 2)}}{2 \pi i} \int_{e^{-\tau} \gamma_{ \pm}}(1-w)^{\Delta t} \frac{d w}{w^{\Delta h+\Delta t / 2+1}}, \quad r \rightarrow+0 .
$$

Now notice that the prefactor $e^{-\tau(\Delta h+\Delta t / 2)}$ will cancel out of any determinant with this kernel, so it can be ignored. We, therefore, make the following

Definition 4. Introduce the following incomplete beta function kernel

$$
\mathrm{B}_{ \pm}(k, l ; z)=\frac{1}{2 \pi i} \int_{\bar{z}}^{z}(1-w)^{k} w^{-l-1} d w,
$$

where the path of the integration crosses $(0,1)$ for the plus sign and $(-\infty, 0)$ for the minus sign.

3.1.8. It is also obvious from our construction that the function $\Re S(z)$ reaches its maximal value on the contour $\gamma_{<}$precisely at the points $z_{c}$ and $\bar{z}_{c}$. The same points are the minima of the function $S(z)$ on the contour $\gamma_{>}$. The behavior of the integral $\int^{(1)}$ is thus determined by the term (29) and, by the basic principle (27), the limit of $\int^{(1)}$ vanishes. 
3.1.9. We can summarize our discussion as follows. Set $z_{*}=e^{-\tau} z_{c}$; in other words,

$$
\left\{z_{*}, \bar{z}_{*}\right\}=\left\{|z|=e^{-\tau / 2}\right\} \cap\left\{|z-1|=e^{-\tau / 4-\chi / 2}\right\}, \quad \Im z_{*}>0 .
$$

It is convenient to extend the meaning of $z_{*}$ to denote the point on the circle $|z|=e^{-\tau / 2}$ which is the closest point to the circle $|z-1|=e^{-\tau / 4-\chi / 2}$ in the case when the two circles do not intersect. In other words, we complement the definition (33) by setting

$$
z_{*}= \begin{cases}e^{-\tau / 2}, & e^{-\chi / 2}<e^{\tau / 4}-e^{-\tau / 4}, \\ -e^{-\tau / 2}, & e^{-\chi / 2}>e^{\tau / 4}+e^{-\tau / 4} .\end{cases}
$$

We have established the following

Theorem 2. Let $U=\left\{\left(t_{i}, h_{i}\right)\right\}$ and suppose that as $r \rightarrow+0$

$$
r t_{i} \rightarrow \tau \geq 0, \quad r h_{i} \rightarrow \chi, \quad i=1,2, \ldots,
$$

in such a way that the differences

$$
\Delta t_{i j}=t_{i}-t_{j}, \quad \Delta h_{i j}=h_{i}-h_{j}
$$

remain fixed. Then, as $r \rightarrow+0$,

$$
\operatorname{Prob}\{U \subset \widetilde{\mathfrak{S}}(\pi)\} \rightarrow \operatorname{det}\left[\mathrm{B}_{ \pm}\left(\Delta t_{i j}, \Delta h_{i j}+\frac{\Delta t_{i j}}{2} ; z_{*}\right)\right],
$$

where the point $z_{*}=z_{*}(\tau, \chi)$ is defined in (33) and (34) and the choice of the plus sign corresponds to $\Delta t_{i j}=t_{i}-t_{j} \geq 0$.

Remark 1. These formulas can be transformed (see Section 3.1.12) into a double integral of the form considered in [5], Proposition 8.5 and Conjecture 13.5.

Remark 2. Observe that the limit correlations are trivial in the cases covered by (34). In other words, they are nontrivial unless the inequality (30) is satisfied. This means that the inequality (30) describes the values of $(\tau, \chi)$ that correspond to the bulk of the limit shape.

3.1.10. In particular, denote by $\rho_{*}(\tau, \chi)$ the limit of 1-point correlation function

$$
K_{3 D}((t, h),(t, h)) \rightarrow \rho_{*}(\tau, \chi), \quad r t \rightarrow \tau, r h \rightarrow \chi .
$$

This is the limiting density of the horizontal tiles at the point $(\tau, \chi)$. We have the following

Corollary 2. The limiting density of horizontal tiles is

$$
\rho_{*}(\tau, \chi)=\frac{\theta_{*}}{\pi},
$$

where $\theta_{*}=\arg z_{*}$ (see Figure [5), that is,

$$
\theta_{*}=\arccos \left(\cosh \frac{\tau}{2}-\frac{e^{-\chi}}{2}\right) .
$$

The level sets of the density as functions of $\tau$ and $\chi$ are plotted in Figure 6 More precisely, Figure 6 shows the curves

$$
\theta_{*}(\tau, \chi)=\frac{k \pi}{8}, \quad k=0, \ldots, 8 .
$$

The knowledge of density $\rho_{*}$ is equivalent to the knowledge of the limit shape; see Sections 3.1.13 and 3.1.14 We point out, however, that additional analysis is needed to prove the convergence to the limit shape in a suitable metric as in [3]. 


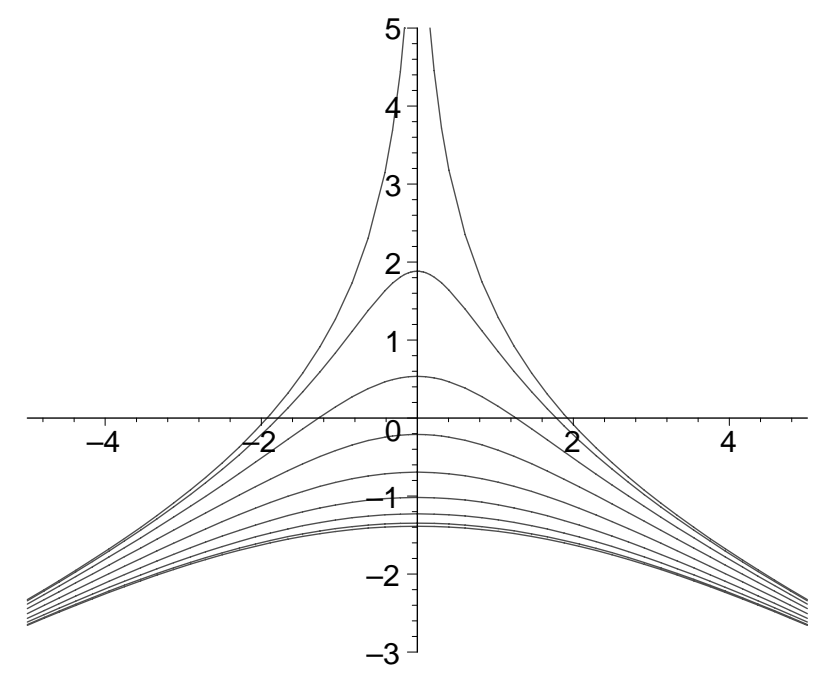

Figure 6. Level sets of the density of horizontal tiles

3.1.11. Corollary 2 can be generalized as follows:

Corollary 3. The equal time correlations are given by the discrete sine kernel; that is, if $t_{1}=t_{2}=\ldots$, then

$$
\operatorname{Prob}\{U \subset \widetilde{\mathfrak{S}}(\pi)\} \rightarrow \operatorname{det}\left[\frac{\sin \left(\theta_{*}\left(h_{i}-h_{j}\right)\right)}{\pi\left(h_{i}-h_{j}\right)}\right],
$$

as $r \rightarrow+0$.

3.1.12. The incomplete beta kernel $\mathrm{B}_{ \pm}$can be transformed into a double integral of the form considered in [5] as follows.

Using the following standard integral

$$
\frac{1}{2 \pi i} \int_{|z|=\alpha} \frac{z^{-k-1} d z}{1-\beta z}= \begin{cases}\beta^{k}, & k \geq 0,|\alpha \beta|<1, \\ -\beta^{k}, & k<0,|\alpha \beta|>1, \\ 0, & \text { otherwise, }\end{cases}
$$

we can replace the condition

$$
|w-1| \lessgtr e^{-\tau / 4-\chi / 2},
$$

in the definition of $\mathrm{B}_{ \pm}\left(k, l ; z_{*}\right)$ by an extra integral. We obtain

$$
\mathrm{B}_{ \pm}\left(k, l ; z_{*}\right)=\frac{1}{(2 \pi i)^{2}} \iint_{\substack{|w|=e^{-\tau / 2} \\|z|=e^{\tau / 4+\chi / 2}}} \frac{z^{-k-1} w^{-l-1}}{1-z+z w} d z d w,
$$

where the plus sign corresponds to $k \geq 0$.

3.1.13. Let $z(\tau, \chi)$ denote the $z$-coordinate of the point on the limit shape corresponding to the point $(\tau, \chi)$. This function can be obtained by integrating the density $\rho_{*}(\tau, \chi)$ as follows.

Consider a tiling such as the one in the Figure 3 and the corresponding 3dimensional diagram, which for the tiling in Figure 3 is shown in Figure 1 It is clear that the $z$-coordinate of the face corresponding to a given horizontal tile 
equals the number of holes (that is, positions not occupied by a horizontal tile) below it. It follows that

$$
z(\tau, \chi)=\int_{-\infty}^{\chi}\left(1-\rho_{*}(\tau, s)\right) d s .
$$

Here, of course, the lower limit of integration can be any number between $-\infty$ and the lower boundary of the limit shape given by the equation (31).

Integrating (35), we obtain the formula

$$
z(\tau, \chi)=\frac{1}{\pi} \int_{0}^{\pi-\theta_{*}} \frac{s \sin s d s}{\cos s+\cosh \frac{\tau}{2}} .
$$

This integral can be evaluated in terms of the dilogarithm function. From (25) we can now compute the other two coordinates as follows:

$$
x(\tau, \chi)=z(\tau, \chi)-\chi-\frac{\tau}{2}, \quad y(\tau, \chi)=z(\tau, \chi)-\chi+\frac{\tau}{2},
$$

which gives a parametrization of the limit shape. A plot of the limit shape is shown in Figure 7

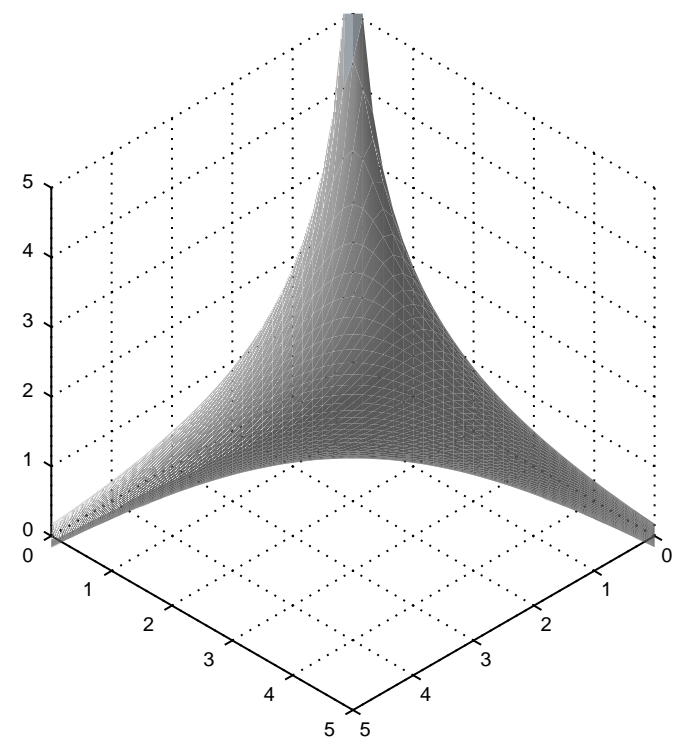

FiguRE 7 . The limit shape

3.1.14. To make the connection to the parametrization of the limit shape given in [3], let us now substitute for $\rho_{*}$ in the integral (37) the formula (36). After a simple coordinate change we obtain

$$
\rho_{*}(\tau, \chi)=\frac{1}{4 \pi^{2}} \iint_{0}^{2 \pi} \frac{d u d v}{1+e^{\chi / 2+\tau / 4+i u}+e^{\chi / 2-\tau / 4+i v}} .
$$

Integrating this in $\chi$, we obtain

$$
z(\tau, \chi)=\frac{1}{2 \pi^{2}} \iint_{0}^{2 \pi} \ln \left|1+e^{\chi / 2+\tau / 4+i u}+e^{\chi / 2-\tau / 4+i v}\right| d u d v .
$$


This together with (39) is equivalent to the following parametrization of the limit shape found in [3]:

$$
(x, y, z)=(f(A, B, C)-2 \ln A, f(A, B, C)-2 \ln B, f(A, B, C)-2 \ln C),
$$

where $A, B, C>0$ and

$$
f(A, B, C)=\frac{1}{2 \pi^{2}} \iint_{0}^{2 \pi} \ln \left|A+B e^{i u}+C e^{i v}\right| d u d v .
$$

This parametrization is manifestly symmetric in $x, y$, and $z$. However, it involves integrals that are more complicated to evaluate than the parametrization (38).

Note that the shape considered in $[3$ differs from ours by a factor of 2 due to different scaling conventions.

\subsection{Universality.}

3.2.1. The reader has surely noticed that all that we really needed for the asymptotics is to be able to deform the contours of integration as in Figure 5 so that the points of the intersection of $\gamma_{>}$and $\gamma_{<}$are the minima of the function $\Re S(z)$ on one curve and the maxima on the other. The intersection points are then forced to be the critical points of the function $S(z)$.

It is clear that this principle is very general and applicable in a potentially very large variety of situations, such as, for example, in the case of Plancherel measure; see below. In particular, the existence of contours of a certain kind is a property which is preserved under small perturbations.

3.2.2. One such perturbation would be to consider anisotropic partitions. The anisotropy in the $t$-direction is especially easy to introduce: one just should replace $q^{|m|}$ in (15) by $q^{V(m)}$ for some function $V$.

3.2.3. Observe that the asymptotics of the equal time correlations are especially easy to obtain in our approach. This is because

$$
\operatorname{Res}_{z=w} \frac{d z}{z-w} \frac{\Phi\left(t_{1}, z\right)}{\Phi\left(t_{2}, w\right)}=1, \quad t_{1}=t_{2},
$$

and hence the analog of the integral $\int^{(2)}$ becomes simply the integral

$$
\int^{(2)}=\frac{1}{2 \pi i} \int_{\gamma_{ \pm}} \frac{d w}{w^{\Delta x+1}}
$$

which leads to the discrete sine kernel in the variable $\Delta x$.

3.2.4. Let us illustrate these general remarks by briefly discussing the asymptotics for the poissonized Plancherel measure [1]. It corresponds to the following specialization of the Schur process:

$$
\phi_{\text {Planch }[}[m]= \begin{cases}e^{\sqrt{\alpha} z}, & m=-\frac{1}{2} \\ e^{\sqrt{\alpha} z^{-1}}, & m=\frac{1}{2} \\ 1, & \text { otherwise }\end{cases}
$$

where $\alpha>0$ is the poissonization parameter. In particular, only the partition $\lambda(0)$ is nontrivial. The correlation kernel at $t=0$ specializes to

$$
K_{\text {Planch }}(x, y)=\frac{1}{(2 \pi i)^{2}} \iint \frac{z^{-x-1 / 2} w^{y-1 / 2}}{z-w} e^{\sqrt{\alpha}\left(z-z^{-1}-w+w^{-1}\right)} d z d w,
$$


which can be expressed in terms of the Bessel functions of the argument $2 \sqrt{\alpha}$; see [1, 8].

3.2.5. The $\alpha \rightarrow \infty$ asymptotics of the kernel $K_{\text {Planch }}$ is easy to obtain from the classical asymptotics of the Bessel functions; see [1]. It is, however, instructive to see how this can be done even more quickly in our framework. Assume that

$$
\frac{x}{\sqrt{\alpha}}, \frac{y}{\sqrt{\alpha}} \rightarrow \xi
$$

in such a way that $\Delta=x-y$ remains fixed. The critical points of the action

$$
S_{\text {Planch }}=z-z^{-1}-\frac{\xi}{2} \ln z
$$

are complex precisely when $|\xi|<2$, in which case they are the points $e^{ \pm i \theta}$, where

$$
\theta=\arccos (\xi / 2) \text {. }
$$

So, the same argument as we employed above immediately yields the following formula from [1]:

$$
K_{\text {Planch }}(x, y) \rightarrow \frac{\sin \theta \Delta}{\pi \Delta}
$$

3.2.6. Of course, a finer analysis (which was carried out in 1) is needed to justify depoissonization in the asymptotics. In our situation, a similar problem is to pass from the $q \rightarrow 1$ asymptotics of the measure $\mathfrak{M}_{q}$ to the asymptotics of the uniform measures on partitions of a given volume $N$ as $N \rightarrow \infty$. In other words, further work is needed to verify the equivalence of ensembles in the asymptotics.

3.2.7. Similarly, finer analysis is needed to work with the asymptotics at the edges of the limit shapes where one expects to see the Airy kernel appear. In the edge scaling, the following equivalent version of the formula (22)

$$
K\left(\left(t_{1}, x_{1}\right),\left(t_{2}, x_{2}\right)\right)=\sum_{m=1 / 2}^{\infty}\left[z^{x_{1}+m} w^{-x_{2}-m}\right] \frac{\Phi\left(t_{1}, z\right)}{\Phi\left(t_{2}, w\right)}
$$

may be helpful because, by analogy to the situation with the Plancherel measure [1, 8], one could expect to see this sum become the integral representing the Airy kernel

$$
\frac{\operatorname{Ai}(x) \operatorname{Ai}^{\prime}(y)-\operatorname{Ai}^{\prime}(x) \operatorname{Ai}(y)}{x-y}=\int_{0}^{\infty} \operatorname{Ai}(x+s) \operatorname{Ai}(y+s) d s .
$$

Further discussion of the Airy-type asymptotics of the integrals of the form (22), (26) can be found in [17.

3.2.8. Recently, the techniques of this paper were used in [7] to prove that, indeed, the boundary of a random 3-dimensional Young diagram converges to the Airy process. 


\section{ApPEndix: Summary of THE INFInIte WEDGE FORMulas}

Let the space $V$ be spanned by $\underline{k}, k \in \mathbb{Z}+\frac{1}{2}$. The space $\Lambda^{\frac{\infty}{2}} V$ is, by definition, spanned by vectors

$$
v_{S}=\underline{s_{1}} \wedge \underline{s_{2}} \wedge \underline{s_{3}} \wedge \ldots,
$$

where $S=\left\{s_{1}>s_{2}>\ldots\right\} \subset \mathbb{Z}+\frac{1}{2}$ is such a subset that both sets

$$
S_{+}=S \backslash\left(\mathbb{Z}_{\leq 0}-\frac{1}{2}\right), \quad S_{-}=\left(\mathbb{Z}_{\leq 0}-\frac{1}{2}\right) \backslash S
$$

are finite. We equip $\Lambda^{\frac{\infty}{2}} V$ with the inner product in which the basis $\left\{v_{S}\right\}$ is orthonormal. In particular, we have the vectors

$$
v_{\lambda}=\underline{\lambda_{1}-\frac{1}{2}} \wedge \underline{\lambda_{2}-\frac{3}{2}} \wedge \underline{\lambda_{4}-\frac{5}{2}} \wedge \ldots,
$$

where $\lambda$ is a partition. The vector

$$
v_{\emptyset}=\underline{-\frac{1}{2}} \wedge-\frac{3}{2} \wedge-\frac{5}{2} \wedge \ldots
$$

is called the vacuum vector.

The operator $\psi_{k}$ is the exterior multiplication by $\underline{k}$,

$$
\psi_{k}(f)=\underline{k} \wedge f .
$$

The operator $\psi_{k}^{*}$ is the adjoint operator. These operators satisfy the canonical anticommutation relations

$$
\psi_{k} \psi_{k}^{*}+\psi_{k}^{*} \psi_{k}=1
$$

all other anticommutators being equal to 0 . We have

$$
\psi_{k} \psi_{k}^{*} v_{S}= \begin{cases}v_{S}, & k \in S, \\ 0, & k \notin S .\end{cases}
$$

The operators $\alpha_{n}$ defined by

$$
\alpha_{n}=\sum_{k \in \mathbb{Z}+\frac{1}{2}} \psi_{k-n} \psi_{k}^{*}, \quad n= \pm 1, \pm 2, \ldots,
$$

satisfy the Heisenberg commutation relations

$$
\left[\alpha_{n}, \alpha_{m}\right]=n \delta_{n,-m}
$$

see the formula. Clearly, $\alpha_{n}^{*}=\alpha_{-n}$. It is clear from definitions that

$$
\left[\alpha_{n}, \psi(z)\right]=z^{n} \psi(z), \quad\left[\alpha_{n}, \psi^{*}(w)\right]=-w^{n} \psi^{*}(w)
$$

and also that

$$
\alpha_{n} v_{\emptyset}=0, \quad n \leq 0 .
$$

\section{ACKNOWLEDGMENTS}

We are grateful to R. Kenyon and A. Vershik for fruitful discussions. The first author was partially supported by NSF grant DMS-0096246 and a Sloan foundation fellowship. The second author was partially supported by NSF grant DMS-0070931. Both authors were partially supported by CRDF grant RM1-2244. 


\section{REFERENCES}

[1] A. Borodin, A. Okounkov, and G. Olshanski, On asymptotics of the Plancherel measures for symmetric groups, J. Amer. Math. Soc. 13 (2000), no. 3, 481-515. MR 2001g:05103

[2] R. Burton and R. Pemantle, Local characteristics, entropy and limit theorems for spanning trees and domino tilings via transfer-impendances, Ann. Prob. 21 (1993), 1329-1371. MR 94m:60019

[3] R. Cerf and R. Kenyon, The low-temperature expansion of the Wulff crystal in the 3D Ising model, Comm. Math. Phys. 222 (2001), 147-179. MR 2002i:82046

[4] H. Cohn, N. Elkies, and J. Propp, Local statistics for random domino tilings of the Aztec diamond, Duke Math. J. 85 (1996), no. 1, 117-166. MR 97k:52026

[5] H. Cohn, R. Kenyon, and J. Propp, A variational principle for domino tilings, math.CO/0008220, J. Amer. Math. Soc. 14 (2001), 297-346. MR 2002k:82038

[6] L. Faddeev and R. Kashaev, Quantum dilogarithm, hep-th/9310070, Modern Phys. Lett. A 9 (1994), 427-434. MR 95i:11150

[7] P. Ferrari and H. Spohn, Step fluctuations for a faceted crystal, cond-mat/0212456.

[8] K. Johansson, Discrete orthogonal polynomials and the Plancherel measure, math.CO/ 9906120, Ann. of Math (2) 153 (2001), 259-296. MR 2002g:05188

[9] K. Johansson, Universality of the local spacing distribution in certain ensembles of Hermitian Wigner matrices, math.ph/0006020, Comm. Math. Phys. 215 (2001), 683-705. MR 2002j:15024

[10] K. Johansson, Non-intersecting paths, random tilings, and random matrices, math.PR/ 0011250, Probab. Theory Related Fields 123 (2002), 225-280.

[11] V. Kac, Infinite dimensional Lie algebras, Cambridge University Press. MR 87c:17023

[12] S. Karlin and G. McGregor, Coincidence probabilities, Pacific J. Math 9 (1959), 1141-1164. MR 22:5072

[13] R. Kenyon, Local statistics of lattice dimers, Ann. Inst. H. Poincaré, Prob. et Stat. 33 (1997), 591-618. MR 99b:82039

[14] R. Kenyon, The planar dimer model with a boundary: a survey, CRM Monogr. Ser., Vol. 13, Amer. Math. Soc., Providence, RI, 2000, pp. 307-328. MR 2002e:82011

[15] I. G. Macdonald, Symmetric functions and Hall polynomials, Clarendon Press, 1995. MR 96h:05207

[16] A. Okounkov, Infinite wedge and random partitions, Selecta Math., New Ser., 7 (2001), 57-81, math.RT/9907127. MR 2002f:60019

[17] A. Okounkov, Symmetric functions and random partitions, Symmetric functions 2001: Surveys of Developments and Perspectives, edited by S. Fomin, Kluwer Academic Publishers, 2002.

[18] M. Praehofer and H. Spohn, Scale Invariance of the PNG Droplet and the Airy Process, math.PR/0105240, J. Statist. Phys. 108 (2002), 1071-1106.

[19] A. Vershik, talk at the 1997 conference on Formal Power Series and Algebraic Combinatorics, Vienna.

Department of Mathematics, University of California at Berkeley, Evans Hall \#3840, Berkeley, California 94720-3840

E-mail address: okounkov@math. berkeley.edu

Department of Mathematics, University of California at Berkeley, Evans Hall \#3840, Berkeley, California 94720-3840

E-mail address: reshetik@math.berkeley.edu 